\title{
Frequency domain conditions for the existence of Bohr almost periodic solutions in evolution equations
}

\author{
Volker Reitmann \\ St. Petersburg State University, \\ Universitetsky prospekt 28, Petrodvorets, \\ St. Petersburg, 198504, RUSSIA \\ VReitmann@aol.com
}

January 22, 2007 - DRAFT

\begin{abstract}
We consider a control problem for the heating process of an elastic plate. The heat flux within the plate is modeled by the heat equation with nonlinear Neumann boundary conditions according to Newton's law. As input at a part of the boundary we take the nonlinearly transformed and modulated heat production of a separate heater which is given by a nonlinear Duffing-type ODE. This ODE depends on measurements of the temperature within the plate and on Bohr resp. Stepanov almost periodic in time forcing terms. The physical problem is generalized to a bifurcation problem for non-autonomous evolution systems in rigged Hilbert spaces. Using Lyapunov functionals, invariant cones and monotonicity properties of the nonlinearities in certain Sobolev spaces, we derive frequency domain conditions for the existence and uniqueness of an asymptotically stable and almost periodic in time temperature field.
\end{abstract}

\section{INTRODUCTION}

Let us introduce some function spaces. We follow the representation in [10]. Suppose $\left(E,\|\cdot\|_{E}\right)$ is a Banach space.

If $J \subset \mathbb{R}$ is an interval, denote by $C(J ; E)$ the space of all continuous functions from $J$ to $E$, endowed with the topology of uniform convergence on compact sets. If $J=\mathbb{R}$ or $J=\mathbb{R}_{+}$the space $C_{b}(J ; E)$ is the subspace of $C(J ; E)$ of bounded functions equipped with the norm

$$
\|f\|_{C_{b}}:=\sup _{u \in J}\|f(u)\|_{E} .
$$

The Banach space of Stepanov bounded on $J=\mathbb{R}$ or $J=$ $\mathbb{R}_{+}$functions (of exponent $p=2$ ) is the space $B S^{2}(J ; E)$ which consists of all functions $f \in L_{\text {loc }}^{2}(J ; E)$ having finite norm

$$
\|f\|_{S^{2}}^{2}:=\sup _{t \in J} \int_{t}^{t+1}\|f(\tau)\|_{E}^{2} d \tau .
$$

A subset $\mathcal{S} \subset \mathbb{R}$ is relatively dense if there is a compact interval $\mathcal{K} \subset \mathbb{R}$ such that $(s+\mathcal{K}) \cap \mathcal{S} \neq \varnothing$ for all $s \in \mathbb{R}$. A function $f \in C_{b}(\mathbb{R} ; E)$ is said to be Bohr almost periodic if for any $\varepsilon>0$ the set

$$
\left\{\tau \in \mathbb{R} \mid \sup _{s \in \mathbb{R}}\|f(s+\tau)-f(s)\| \leq \varepsilon\right\}
$$

of $\varepsilon$-almost periods is relatively dense in $\mathbb{R}$.

For a function $f \in L_{\text {loc }}^{2}(\mathbb{R} ; E)$, put

$$
f^{b}(t):=f(t+w), w \in[0,1], t \in \mathbb{R} .
$$

The function $f^{b}(t)$ is regarded as a function with values in the space $L^{2}(0,1 ; E)$. Then

$$
B S^{2}(\mathbb{R} ; E)=\left\{f \in L_{\mathrm{loc}}^{2}(\mathbb{R} ; E) \mid f^{b} \in L^{\infty}\left(\mathbb{R} ; L^{2}(0,1 ; E)\right)\right\}
$$

and, moreover, $\|f\|_{S^{2}}=\left\|f^{b}\right\|_{L^{\infty}}$.

A function $f \in B S^{2}(\mathbb{R} ; E)$ is called an almost periodic function in the sense of Stepanov and of exponent 2 (abbreviated $S^{2}$-a.p.) if $f^{b} \in \operatorname{CAP}\left(\mathbb{R} ; L^{2}(0,1 ; E)\right)$. In this case the $\varepsilon$-almost periods of $f^{b}$ are called the $\varepsilon$-almost periods of $f$. The space of $S^{2}$-a.p. functions with values in $E$ is denoted by $S^{2}(\mathbb{R} ; E)$. Obviously, CAP $(\mathbb{R} ; E) \subset S^{2}(\mathbb{R} ; E)$.

\section{Control systems in LuR'e form With a DufFing TYPE NONLINEARITY}

Let $\mathcal{V}_{1} \subset \mathcal{V}_{0} \subset \mathcal{V}_{-1}$ be a Gelfand rigging of the real Hilbert space $\mathcal{V}_{0}$, i.e. a chain of Hilbert spaces with dense and continuous inclusions. Denote by $(\cdot, \cdot) \mathcal{V}_{j}$ and $\|\cdot\|_{\mathcal{V}_{j}}, j=$ $1,0,-1$, the scalar product resp. norm in $\mathcal{V}_{j}(j=1,0,-1)$ and by $(\cdot, \cdot) \mathcal{V}_{-1}, \mathcal{V}_{1}$ the pairing between $\mathcal{V}_{-1}$ and $\mathcal{V}_{1}$.

Let $A_{0} \in \mathcal{L}\left(\mathcal{V}_{1}, \mathcal{V}_{-1}\right)$ be a linear operator, $b_{0} \in \mathcal{V}_{-1}$ a generalized vector, $c_{0} \in \mathcal{V}_{0}$ a vector and $d_{0}<0$ a number. According to the vectors $c_{0}$ and $b_{0}$ we introduce the linear operators $C_{0} \in \mathcal{L}\left(\mathcal{V}_{0}, \mathbb{R}\right)$ and $B_{0} \in \mathcal{L}\left(\mathbb{R}, \mathcal{V}_{-1}\right)$ by $C_{0} \nu=\left(c_{0}, \nu\right)_{\mathcal{V}_{0}}, \forall \nu \in \mathcal{V}_{0}$, and $B_{0} \xi:=\xi b_{0}, \forall \xi \in \mathbb{R}$.

Assume that $\phi: \mathbb{R} \times \mathbb{R} \rightarrow \mathbb{R}$ and $g: \mathbb{R} \rightarrow \mathbb{R}$ are two scalarvalued functions. Our aim is to study a system of indirect control, which is formally given as

$$
\begin{aligned}
& \dot{\nu}=A_{0} \nu+b_{0}[\phi(t, z)+g(t)], \\
& \dot{z}=\left(c_{0}, \nu\right)_{\mathcal{V}_{0}}+d_{0}[\phi(t, z)+g(t)] .
\end{aligned}
$$

Let us demonstrate how (1) can be written as a standard control system. Consider for this the Gelfand rigging $Y_{1} \subset$ $Y_{0} \subset Y_{-1}$, in which

$$
Y_{j}:=\mathcal{V}_{j} \times \mathbb{R}, \quad j=1,0,-1 .
$$

The scalar product $(\cdot, \cdot)_{j}$ in $Y_{j}$ is introduced as $\left(\left(\nu_{1}, z_{1}\right),\left(\nu_{2}, z_{2}\right)\right)_{j}:=\left(\nu_{1}, \nu_{2}\right) \mathcal{\nu}_{j}+z_{1} z_{2}$, where $\left(\nu_{1}, z_{1}\right),\left(\nu_{2}, z_{2}\right) \in Y_{j}$ are arbitrary. The pairing between $Y_{-1}$ and $Y_{1}$ is defined for $(h, \xi) \in \mathcal{V}_{-1} \times \mathbb{R}=Y_{-1}$ and $(\nu, \varsigma) \in \mathcal{V}_{1} \times \mathbb{R}=Y_{1}$ through

$$
((h, \xi),(\nu, \varsigma))_{-1,1}:=(h, \nu)_{\mathcal{V}_{-1}, \mathcal{V}_{1}}+\xi \varsigma .
$$


Let $b:=\left[\begin{array}{l}b_{0} \\ d_{0}\end{array}\right] \in Y_{-1}$ and $c:=\left[\begin{array}{l}0 \\ 1\end{array}\right] \in Y_{0}$. Suppose further that the operators $C \in \mathcal{L}\left(Y_{0}, \mathbb{R}\right)$ and $B \in \mathcal{L}\left(\mathbb{R}, Y_{-1}\right)$ are given as

$$
C y=(c, y)_{0}, \quad \forall y \in Y_{0}, \quad B \xi=\xi b, \quad \forall \xi \in \mathbb{R},
$$

and the operator $A \in \mathcal{L}\left(Y_{1}, Y_{-1}\right)$ is defined as

$$
A:=\left[\begin{array}{ll}
A_{0} & 0 \\
C_{0} & 0
\end{array}\right] \text {. }
$$

Consider now the system

$$
\dot{y}=A y+B[\phi(t, z)+g(t)], z=C y,
$$

which is equivalent to (1) through $y=(\nu, z)$. If $-\infty \leq T_{1}<$ $T_{2} \leq+\infty$ are arbitrary, we define the norm for Bochner measurable functions in $L^{2}\left(T_{1}, T_{2} ; Y_{j}\right), j=1,0,-1$, by

$$
\|y\|_{2, j}:=\left(\int_{T_{1}}^{T_{2}}\|y(t)\|_{j}^{2} d t\right)^{1 / 2}
$$

Let $\mathcal{W}\left(T_{1}, T_{2} ; Y_{1}, Y_{-1}\right)$ be the space of functions $y$ such that $y \in L^{2}\left(T_{1}, T_{2} ; Y_{1}\right)$ and $\dot{y} \in L^{2}\left(T_{1}, T_{2} ; Y_{-1}\right)$, equipped with the norm

$$
\|y\|_{\mathcal{W}\left(T_{1}, T_{2} ; Y_{1}, Y_{-1}\right)}:=\left(\|y\|_{2,-1}^{2}+\|\dot{y}\|_{2,-1}^{2}\right)^{1 / 2} .
$$

Let us introduce the following assumptions (A1) - (A6) about the operator $A_{0} \in \mathcal{L}\left(\mathcal{V}_{1}, \mathcal{V}_{-1}\right)$, the vectors $b_{0} \in \mathcal{V}_{-1}$ and $c_{0} \in \mathcal{V}_{0}$, and the functions $\phi$ and $g$.

(A1) For any $T>0$ and any $\left(f_{1}, f_{2}\right) \in L^{2}\left(0, T ; \mathcal{V}_{-1} \times \mathbb{R}\right)$ the problem

$$
\begin{aligned}
& \dot{\nu}=A_{0} \nu+f_{1}(t), \\
& \dot{z}=\left(c_{0}, \nu\right)_{\mathcal{V}_{0}}+f_{2}(t), \quad(\nu(0), z(0))=\left(\nu_{0}, z_{0}\right)
\end{aligned}
$$

is well-posed, i.e. for arbitrary $\left(\nu_{0}, z_{0}\right) \in Y_{0},\left(f_{1}, f_{2}\right) \in$ $L^{2}\left(0, T ; \mathcal{V}_{-1} \times \mathbb{R}\right)$ there exists a unique solution $(\nu, z) \in$ $\mathcal{W}\left(0, T ; Y_{1}, Y_{-1}\right)$ satisfying (7) in a variational sense and depending continuously on the initial data, i.e.

$$
\begin{aligned}
& \|(\nu, z)\|_{\mathcal{W}\left(0, T ; Y_{1}, Y_{-1}\right)}^{2} \leq \\
& k_{1}\left\|\left(\nu_{0}, z_{0}\right)\right\|_{\mathcal{V}_{0} \times \mathbb{R}}^{2}+k_{2}\left\|\left(f_{1}, f_{2}\right)\right\|_{2,-1}^{2},
\end{aligned}
$$

where $k_{1}>0$ and $k_{2}>0$ are some constants .

(A2) There is a $\lambda>0$ such that $A_{0}+\lambda I$ is a Hurwitz operator .

(A3) For any $T>0,\left(\nu_{0}, z_{0}\right) \in \mathcal{V}_{1} \times \mathbb{R},\left(\tilde{\nu}_{0}, \tilde{z}_{0}\right) \in \mathcal{V}_{1} \times \mathbb{R}$ and $\left(f_{1}, f_{2}\right) \in L^{2}\left(0, T ; \mathcal{V}_{1} \times \mathbb{R}\right)$ the solution of the direct problem (7) and the solution of the adjoint problem

$$
\begin{aligned}
& \dot{\tilde{\nu}}=-\left(A_{0}^{+}+\lambda I\right) \tilde{\nu}+f_{1}(t) \\
& \dot{\tilde{z}}=-C_{0}^{+} \tilde{z}-\lambda \tilde{z}+f_{2}(t)
\end{aligned}
$$

are strongly continuous in $t$ in the norm of $\mathcal{V}_{1} \times \mathbb{R}$.

(A4) The pair $\left(A_{0}, b_{0}\right)$ is $L^{2}$-controllable, i.e. for arbitrary $\nu_{0} \in \mathcal{V}_{0}$ there exists a control $\xi(\cdot) \in L^{2}(0, \infty ; \mathbb{R})$ such that the problem

$$
\dot{\nu}=A_{0} \nu+b_{0} \xi, \quad \nu(0)=\nu_{0}
$$

is well-posed in the variational sense on $(0, \infty)$.

Introduce by ( $c$ denotes the complexification)

$$
\chi(p)=\left(c_{0}^{c},\left(A_{0}^{c}-p I^{c}\right)^{-1} b_{0}^{c}\right)_{\mathcal{V}_{0}}, \quad p \in \rho\left(A_{0}^{c}\right)
$$

the transfer function of the triple $\left(A_{0}^{c}, b_{0}^{c}, c_{0}^{c}\right)$.

(A5) Suppose $\lambda>0$ and $\kappa_{1}>0$ are parameters, where $\lambda$ is from (A2). Then:

$$
\begin{aligned}
& \text { a) } \lambda d_{0}+\operatorname{Re}(-i \omega-\lambda) \chi(i \omega-\lambda)+ \\
& \kappa_{1}\left|\chi(i \omega-\lambda)-d_{0}\right|^{2} \leq 0, \quad \forall \omega \geq 0 .
\end{aligned}
$$

(A6) The function $\phi: \mathbb{R} \times \mathbb{R} \rightarrow \mathbb{R}$ is continuous and $\phi(t, 0)=$ $0, \forall t \in \mathbb{R}$. The function $g: \mathbb{R} \rightarrow \mathbb{R}$ belongs to $L_{\text {loc }}^{2}(\mathbb{R} ; \mathbb{R})$. There are numbers $\kappa_{1}>0$ (from (A5)), $0 \leq \kappa_{2}<\kappa_{3}<$ $+\infty, \beta_{1}<\beta_{2}$ and $\zeta_{2}<\zeta_{1}$ such that:

$$
\text { a) } \beta_{1}<g(t)<\beta_{2} \text {, }
$$

for a.a. $\mathrm{t}$ from an arbitrary compact time interval ;

$$
\begin{array}{r}
\left(\phi(t, z)+\beta_{i}\right)\left(z-\zeta_{i}\right) \leq \kappa_{1}\left(z-\zeta_{i}\right)^{2}, \quad i=1,2, \\
\forall t \in \mathbb{R}, \quad \forall z \in\left[\zeta_{2}, \zeta_{1}\right] ; \quad \\
\text { c) } \quad \kappa_{2}\left(z_{1}-z_{2}\right)^{2} \leq\left(\phi\left(t, z_{1}\right)-\phi\left(t, z_{2}\right)\right)\left(z_{1}-z_{2}\right) \leq \\
\kappa_{3}\left(z_{1}-z_{2}\right)^{2}, \quad \forall t \in \mathbb{R}, \forall z_{1}, z_{2} \in\left[\zeta_{2}, \zeta_{1}\right] .
\end{array}
$$

We assume in the next theorem that the solutions of (1) are for every $T>0$ elements of the space $\mathcal{W}\left(0, T ; Y_{1}, Y_{-1}\right)$. Then we show the existence of solutions with initial states from a certain set.

Theorem 1: Assume that for system (1) the hypotheses (A1) - (A6) are satisfied. Then there exists a closed, positively invariant and convex set $\mathcal{G}$ such that

$$
\begin{aligned}
& \left\{(\nu, z) \in \mathcal{V}_{1} \times \mathbb{R} \mid \nu=0, z \in\left[\zeta_{2}, \zeta_{1}\right]\right\} \subset \mathcal{G} \subset \\
& \left\{(\nu, z) \in \mathcal{V}_{1} \times \mathbb{R} \mid z \in\left[\zeta_{2}, \zeta_{1}\right]\right\} .
\end{aligned}
$$

In order to prove this Theorem we need some auxilary results.

Suppose that $Y_{1} \subset Y_{0} \subset Y_{-1}$ is a Gelfand rigging of $Y_{0}$, $\|\cdot\|_{j},(\cdot, \cdot)_{j}$ are the corresponding norms and scalar products, respectively, and $(\cdot, \cdot)_{-1,1}$ is the pairing between $Y_{-1}$ and $Y_{1}$. Consider the linear system

$$
\dot{y}=A y, \quad z=(c, y)_{0},
$$

where $A \in \mathcal{L}\left(Y_{1}, Y_{-1}\right)$ and $c \in Y_{0}$.

Assume that for each $y_{0} \in Y_{0}$ there exists a unique solution $y\left(\cdot, y_{0}\right)$ of $(13)$ in $\mathcal{W}(0, \infty)$ satisfying $y\left(0, y_{0}\right)=y_{0}$. In the sequel we need the following assumption ([2]).

(A7) The space $Y_{0}$ can be decomposed as $Y_{0}=Y_{0}^{+} \oplus Y_{0}^{-}$ such that the following holds:

a) For each $y_{0} \in Y_{0}^{+}$we have $\lim _{t \rightarrow \infty} y\left(t, y_{0}\right)=0$. For each $y_{0} \in Y_{0}^{-}$there exists a unique solution $y_{-}(t)=y\left(t, y_{0}\right)$ of $(13)$, defined on $(-\infty, 0)$, such 
that $\lim _{t \rightarrow-\infty} y_{-}(t)=0$ and $\left(c, y\left(t, y_{0}\right)\right)_{0}=0, \forall t \geq 0$, if and only if $y_{0}=0$.

b) For each $y_{0} \in Y_{0}^{+}$the equality $\left(c, y\left(t, y_{0}\right)\right)_{0}=0$, $\forall t \leq 0$, holds if and only if $y_{0}=0$. For each $y_{0} \in Y_{0}^{-}$ the equality $\left(c, y\left(t, y_{0}\right)\right)_{0}=0, \forall t \leq 0$, holds if and only if $y_{0}=0$.

Remark 1: Assumption (A7) a) means that we assume for the linear system (13) the decomposition of $Y_{0}$ in $y=0$ into a stable subspace $E^{s} \equiv Y_{0}^{+}$and an unstable subspace $E^{u} \equiv$ $Y_{0}^{-}$. Assumption (A7) b) characterizes the identifiability in the sense of Kalman of the pair $(A, c)$ on $Y_{0}^{+}$and $Y_{0}^{-}$, respectively.

In the following $L \geq 0$ for a linear operator $L \in \mathcal{L}(Y), Y$ a Hilbert space, means that $L$ is positive, i.e. $(y, L y)_{Y}>0$, $\forall y \in Y \backslash\{0\} ; \quad L \leq 0$ means that $-L$ is positive.

Lemma 1: Suppose that system (13) satisfies (A7) and there exists a linear continuous operator $P: Y_{0} \rightarrow Y_{0}, P^{*}=P$, such that for any $s \leq t$ and any solution $y\left(\cdot, y_{0}\right)$ of (13) we have with $V(y):=(y, P y)_{0}, y \in Y_{0}$,

$$
V\left(y\left(t, y_{0}\right)\right)-V\left(y\left(s, y_{0}\right)\right) \leq-\int_{s}^{t}\left(c, y\left(\tau, y_{0}\right)\right)_{0}^{2} d \tau \text {. }
$$

Then

$$
P_{\mid Y_{0}^{+}} \geq 0 \text {, i.e., }(y, P y)_{0}>0 \text { for all } y \in Y_{0}^{+} \backslash\{0\}
$$

and

$$
P_{\mid Y_{0}^{-}} \leq 0 \text {, i.e., }(y, P y)_{0}<0 \text { for all } y \in Y_{0}^{-} \backslash\{0\} .
$$

Proof: Let $y_{0} \in Y_{0}^{+} \backslash\{0\}$. Then by (A7) a) we have $\lim _{t \rightarrow \infty} y\left(t, y_{0}\right)=0$ and, due to the boundedness of $P$, $\lim _{t \rightarrow \infty} V\left(y\left(t, y_{0}\right)\right)=0$. It follows from (14) for $s=0$ and $t \rightarrow \infty$ that

$$
-V\left(y_{0}\right) \leq-\int_{0}^{\infty}\left(c, y\left(\tau, y_{0}\right)\right)_{0}^{2} d \tau
$$

Using again (A7) a), we conclude from (17) that

$$
V\left(y_{0}\right) \geq \int_{0}^{\infty}\left(c, y\left(\tau, y_{0}\right)\right)_{0}^{2} d \tau>0 \text {. }
$$

Thus (15) is shown.

Let now $y_{0} \in Y_{0}^{-} \backslash\{0\}$. Then by (A7) b) we have $\lim _{t \rightarrow-\infty} y\left(t, y_{0}\right)=0$ and, consequently,

$\lim _{t \rightarrow-\infty} V\left(y\left(t, y_{0}\right)\right)=0$. If we take in (14) $s \rightarrow-\infty$ and $t \rightarrow 0$, we receive

$$
V\left(y_{0}\right) \leq-\int_{-\infty}^{0}\left(c, y\left(\tau, y_{0}\right)\right)_{0}^{2} d \tau
$$

Assumption (A1) b) implies that $\int_{-\infty}^{0}\left(c, y\left(\tau, y_{0}\right)\right)_{0}^{2} d \tau>0$. Thus we conclude from (18) that $V\left(y_{0}\right)<0$. This proves (16).

The next lemma is concerned with the separation of quadratic cones by special functionals. Let us recall some definitions. Assume that $Y$ is a Hilbert space with scalar product $(\cdot, \cdot)$.
A cone in $Y$ is a set $\mathcal{C} \subset Y, \mathcal{C} \neq \varnothing$, such that $y \in \mathcal{C}, \alpha \in \mathbb{R}_{+}$ imply that $\alpha y \in \mathcal{C}$. It is easy to see that a cone $\mathcal{C}$ in $Y$ is convex if and only if $y_{1}, y_{2} \in \mathcal{C}$ imply that $y_{1}+y_{2} \in \mathcal{C}$.

Suppose that $P \in \mathcal{L}(Y), P=P^{*}$. Then the set $\mathcal{C}:=\{y \in$ $Y \mid(y, P y) \leq 0\}$ is a cone which is called by us quadratic.

Assume that there is a decomposition $Y=Y^{+} \oplus Y^{-}$such that $P_{\mid Y^{+}} \geq 0$ and $P_{\mid Y^{-}} \leq 0$. Then the quadratic cone $\{y \in Y \mid(y, P y) \leq 0\}$ is called by us quadratic cone of dimension $\operatorname{dim} Y^{-}$.

\section{Lemma 2: Suppose that:}

1) $Y_{1} \subset Y_{0} \subset Y_{-1}$ is a Gelfand rigging of the Hilbert space $Y_{0}$ with scalar products $(\cdot, \cdot)_{i}$, corresponding norms $\|\cdot\|_{i}, i=1,0,-1$, and pairing $(\cdot, \cdot)_{-1}$, between $Y_{-1}$ and $Y_{1}$;

2) There is an operator $P \in \mathcal{L}\left(Y_{-1}, Y_{0}\right) \cap \mathcal{L}\left(Y_{0}, Y_{1}\right)$, selfadjoint and positive in $Y_{0}$ such that

$$
\mathcal{C}:=\left\{y \in Y_{0} \mid(y, P y)_{0} \leq 0\right\}
$$

is an 1-dimensional quadratic cone;

3) There are vectors $h \in Y_{-1}$ and $r \in Y_{0}$ such that

$$
\begin{aligned}
& 2(h, P y)_{-1,1}=(r, y)_{0}, \forall y \in Y_{1} \\
& \text { and } \quad(h, r)_{-1,1}<0 \text {. }
\end{aligned}
$$

Then we have

$$
\operatorname{int} \mathcal{C} \cap\left\{y \in Y_{1} \mid(y, r)_{0}=0\right\}=\varnothing .
$$

Proof: Suppose that (21) is not true, i.e., assume that there is a $y_{0} \in Y_{1}, y_{0} \neq 0$, such that

$$
\left(y_{0}, P y_{0}\right)_{0}<0 \quad \text { and } \quad\left(y_{0}, r\right)_{0}=0 .
$$

Since $\mathcal{C}$ is a cone, we have $\alpha y_{0} \in \mathcal{C}, \forall \alpha \in \mathbb{R}$, and

$$
\operatorname{span}\left\{y_{0}\right\} \backslash\{0\} \subset \operatorname{int} \mathcal{C} .
$$

Since the inclusions $Y_{1} \subset Y_{0} \subset Y_{-1}$ are dense, there exists a sequence $\left\{h_{n}\right\}_{n=1}^{\infty}, h_{n} \in Y_{1}(n=1,2, \ldots)$ such that $h_{n} \rightarrow$ $h$ for $n \rightarrow \infty$ in the norm of $Y_{-1}$.

Because of (19) we have

$$
2\left(h_{n}, P h_{n}\right)_{0} \rightarrow\left(r, h_{n}\right)_{0} \quad \text { for } \quad n \rightarrow \infty .
$$

Since $(\cdot, \cdot)_{-1,1}$ is the unique extension by continuity of the scalar product $(\cdot, \cdot)_{0}$ defined on $Y_{0} \times Y_{1}$, it follows from (20) that there are numbers $\varepsilon_{0}>0$ and $n_{0} \in \mathbb{N}$ such that

$$
\left(r, h_{n}\right)_{0} \leq-\varepsilon_{0}<0, \quad \forall n \geq n_{0} .
$$

Thus for each $\varepsilon_{1} \in\left(0, \varepsilon_{0}\right)$ there is an $n_{1} \in \mathbb{N}$ such that

$$
4\left(h_{n}, P h_{n}\right)_{0} \leq-\varepsilon_{1}^{\prime}, \quad \forall n \geq n_{1},
$$

where $\varepsilon_{1}^{\prime}:=\nu-\varepsilon_{1}$.

From (19) we conclude that $2\left(h_{n}, P y_{0}\right)_{0} \rightarrow\left(r, y_{0}\right)_{0}=0$ for $n \rightarrow \infty$. Thus we have for each $\varepsilon_{2}>0$ a number $n_{2} \in \mathbb{N}$ such that

$$
2\left|\left(h_{n}, P y_{0}\right)_{0}\right|<\varepsilon_{2}, \quad \forall n \geq n_{2} .
$$

Take now $\bar{n}:=\max \left\{n_{0}, n_{1}, n_{2}\right\}$. Then the properties (24) - (27) are satisfied for $n \geq \bar{n}$. By (22) and the inequality 
$\left(\varepsilon_{0}-\varepsilon_{1}\right)>0$, we can choose the number $\varepsilon_{2}$ in (27) so small that

$$
-\left(y_{0}, P y_{0}\right)_{0}\left(\varepsilon_{0}-\varepsilon_{1}\right)-\varepsilon_{2}^{2}>0 .
$$

Let us show now that the plane $\Pi:=\left\{\alpha y_{0}+\beta 2 h_{\bar{n}} \mid \alpha, \beta \in\right.$ $\mathbb{R}\}$, with exception of the point 0 , is contained in $\operatorname{int} \mathcal{C}$. This will be a contradiction to assumption 2) of the theorem if we show that $\operatorname{dim} \Pi=2$. Suppose that this is not the case. This means that there is a $\alpha_{0} \neq 0$ such that

$$
\alpha_{0} y_{0}=h_{\bar{n}} .
$$

It follows from (25) and (29) that $\left(r, h_{\bar{n}}\right)_{0}<0$, and from (22) and (30) that $\left(r, h_{\bar{n}}\right)_{0}=0$. This contradiction shows that $\operatorname{dim} \Pi=2$. It remains to demonstrate that $\Pi \backslash\{0\} \subset$ $\operatorname{int} \mathcal{C}$. Consider for arbitrary $\alpha, \beta \in \mathbb{R}$ with $\alpha^{2}+\beta^{2}>0$ the expression

$$
\begin{aligned}
& \left(\alpha y_{0}+\beta 2 h_{\bar{n}}, P\left(\alpha y_{0}+\beta 2 h_{\bar{n}}\right)\right)_{0} \\
& =\alpha^{2}\left(y_{0}, P y_{0}\right)_{0}+4 \alpha \beta\left(h_{\bar{n}}, P y_{0}\right)_{0}+\beta^{2} 4\left(h_{\bar{n}}, P h_{\bar{n}}\right)_{0} .
\end{aligned}
$$

Under our conditions the quadratic form (30) is negative definite. Really, from (22) we have $\left(y_{0}, P y_{0}\right)_{0}<0$ and from (26) $4\left(h_{\bar{n}}, P h_{\bar{n}}\right)_{0}<0$. Thus by the Routh criterion the negative definiteness of the form is shown if the determinant $D$, associated to this form, is positive. The straight forward computation of $D$ and the use of (26) - (28) gives the estimates

$$
\begin{aligned}
D & =\left(y_{0}, P y_{0}\right)_{0} 4\left(h_{\bar{n}}, P h_{\bar{n}}\right)_{0}-\left(4 h_{\bar{n}}, P y_{0}\right)_{0}^{2} \\
& \geq-\left(y_{0}, P y_{0}\right)_{0}\left(\varepsilon_{0}-\varepsilon_{1}\right)-\varepsilon_{2}^{2}>0 .
\end{aligned}
$$

Remark 2: Lemma 2 can be considered as generalized lemma about the separation of cones ([1], [3], [8], [12]). Really, in the finite-dimensional case we have $Y_{1}=Y_{0}=$ $Y_{-1}=\mathbb{R}^{n},(\cdot, \cdot)_{-1,1}=(\cdot, \cdot)_{0}=(\cdot, \cdot)$ the Euclidean inner product and $P=P^{*}$, det $P \neq 0$, a regular symmetric $n \times n$ matrix. Assumption (19) in Lemma 2 states that there are vectors $h, r \in \mathbb{R}^{n}$ such that

$$
2(h, P y)=(r, y), \quad \forall y \in \mathbb{R}^{n} .
$$

It follows from (31) that

$$
2 h=P^{-1} r .
$$

Equation (32) shows that assumption (20) of Lemma 2 takes the form

$$
\left(r, P^{-1} r\right)<0 .
$$

If (33) is satisfied, it follows from Lemma 2 for the 1dimensional quadratic cone $\mathcal{C}=\left\{y \in \mathbb{R}^{n} \mid(y, P y) \leq 0\right\}$ that

$$
\operatorname{int} \mathcal{C} \cap\left\{y \in \mathbb{R}^{n} \mid(y, r)=0\right\}=\varnothing .
$$

But this is exactly the sufficient part of the statement in [3].

Now we prove for (1) the existence of solutions in $\mathcal{W}\left(0, T ; \mathcal{V}_{1} \times \mathbb{R}, \mathcal{V}_{-1} \times \mathbb{R}\right)$ and the existence of at least one solution in $C_{b}\left(\mathbb{R} ; \mathcal{V}_{0} \times \mathbb{R}\right) \cap B S^{2}\left(\mathbb{R} ; \mathcal{V}_{1} \times \mathbb{R}\right)$. We need for this the a priori inclusion given by Theorem 1 and two additional assumptions.

(A8) The imbedding $\mathcal{V}_{1} \subset \mathcal{V}_{0}$ is compact.

(A9) The family of operators $\{\mathcal{A}(t)\}_{t \in \mathbb{R}}, \mathcal{A}(t): Y_{1} \rightarrow Y_{-1}$, given by $\mathcal{A}(t) \eta:=-A \eta-B \phi(t, C \eta), \forall t \in \mathbb{R}, \forall \eta \in Y_{1}$, is monotone on the segment $\left\{\eta \in Y_{1} \mid C \eta \in\left[\zeta_{2}, \zeta_{1}\right]\right\}$, i.e. for any $t \in \mathbb{R}$ we have

$$
\begin{aligned}
& (\mathcal{A}(t) \eta-\mathcal{A}(t) \vartheta, \eta-\vartheta)_{-1,1} \geq 0, \\
& \forall \eta, \vartheta \in Y_{1}, \quad \text { such that } \quad C \eta, C \vartheta \in\left[\zeta_{2}, \zeta_{1}\right] .
\end{aligned}
$$

There exists a continuous function $\tilde{\phi}: \mathbb{R} \times \mathbb{R}$ such that $\tilde{\phi}_{\mid \mathbb{R} \times\left[\zeta_{2}, \zeta_{1}\right]}=\phi$ and (35) with $\tilde{\phi}$ instead of $\phi$ is satisfied for all $\eta, \vartheta \in Y_{1}$.

Remark 3: If $\phi$ has the form $\phi(t, z)=\phi_{1}(t) \phi_{2}(z)$ with $\phi_{1}$ and $\phi_{2}$ continuous, it is clear that such a monotone extension exists.

Theorem 2: Assume that for system (1) the assumptions (A1) - (A9) are satisfied. Then it holds:

a) For any $g \in B S^{2}(\mathbb{R} ; \mathbb{R})$ and any $\left(\nu_{0}, z_{0}\right) \in \mathcal{G}$, where $\mathcal{G}$ is the associated positively invariant set, there exists a solution $(\nu, z) \in \mathcal{W}\left(0, \infty ; \mathcal{V}_{1} \times \mathbb{R}, \mathcal{V}_{-1} \times \mathbb{R}\right)$ of (1) such that $(\nu(0), z(0))=\left(\nu_{0}, z_{0}\right)$.

b) For any $g \in B S^{2}(\mathbb{R} ; \mathbb{R})$ there exists for (1) a solution

$$
\left(\nu_{*}, z_{*}\right) \in C_{b}\left(\mathbb{R} ; \mathcal{V}_{0} \times \mathbb{R}\right) \cap B S^{2}\left(\mathbb{R} ; \mathcal{V}_{1} \times \mathbb{R}\right)
$$

(A10) Any continuous function $\phi$ which satisfies (11a) and (11b) has a continuous extension to a function $\tilde{\tilde{\phi}}: \mathbb{R} \times \mathbb{R} \rightarrow \mathbb{R}$ which satisfies (11a) and (11b) for all $(t, z) \in \mathbb{R} \times \mathbb{R}$.

The next theorem is a generalization of a result from [1] for evolution systems in rigged Hilbert spaces with Duffing-type nonlinearity.

Theorem 3: Assume that for system (1) the assumptions (A1) - (A10) are satisfied and in addition to this the following holds:

(i) The operator $\left[\begin{array}{cc}A_{0} & \kappa_{2} B_{0} \\ C_{0} & \kappa_{2} d_{0}\end{array}\right]$ from $\mathcal{L}\left(Y_{1}, Y_{-1}\right)$ is Hurwitz;

(ii) There exists a number $\epsilon>0$ such that

$$
\frac{1}{\kappa_{3}-\kappa_{2}}+\operatorname{Re} \frac{\chi(i \omega)-d_{0}}{i \omega+\kappa_{2}\left(\chi(i \omega)-d_{0}\right)}>\epsilon, \forall \omega \in \mathbb{R} .
$$

Then we have:

a) For any $g \in B S^{2}(\mathbb{R} ; \mathbb{R})$ system (1) has a unique solution $\left(\nu_{*}, w_{*}\right)$ inside $\mathcal{G}$ which satisfies (36) and this solution is exponentially stable inside $\mathcal{G}$.

b) Let the families of functions $\left\{\phi(\cdot, z) \mid z \in\left[\zeta_{2}, \zeta_{1}\right]\right\}$ and $\{\tilde{\phi}(\cdot, z) \mid z \in \mathcal{S}\}$, where $\tilde{\phi}$ is from (A9) and $\mathcal{S} \subset \mathbb{R}$ is an arbitrary bounded interval, be uniformly Bohr a.p. . Then for any $S^{2}$-a.p. forcing function $g$ the unique in $\mathcal{G}$ bounded and exponentially stable solution $\left(\nu_{*}, z_{*}\right)$ is Bohr a.p. . 


\section{EXAMPLE}

We consider the restricted boundary control problem for the temperature

$$
\begin{aligned}
& \theta_{t}=\delta_{1} \theta_{x x}-\delta_{2} \theta, \quad \delta_{1}>0, \delta_{2}>0 \\
& \theta_{x_{\mid x=0}}=0, \theta_{x_{\mid x=1}}=\delta_{3}[\phi(t, w)+g(t)], \quad \delta_{3} \in \mathbb{R}, \\
& \dot{w}=\int_{0}^{1} \theta(x, t) k(x) d x+\delta_{4}[\phi(t, w)+g(t)],
\end{aligned}
$$$$
k(\cdot) \text { kernel function, } \delta_{4}<0,
$$$$
\phi(t, w)=w-\delta_{5}(t) w^{3} \quad \text { Duffing-type nonlinearity, }
$$$$
\delta_{5}(t) \geq 0 \text { a.e. }
$$

Write (38), (39) as ODE in Hilbert space

$$
\begin{aligned}
\dot{\nu} & =A_{0} \nu+B_{0}[\phi(t, w)+g(t)] \\
\dot{w} & =C_{0} \nu+d_{0}[\phi(t, w)+g(t)],
\end{aligned}
$$

$\mathcal{V}_{1}:=W^{1,2}(0,1), \quad \mathcal{V}_{0}:=L_{2}(0,1), \quad \mathcal{V}_{-1}=\mathcal{V}_{1}^{*}$,

$$
\text { space of test state space dual space }
$$$$
\text { functions (w.r.t. } \mathcal{V}_{0} \text { ) }
$$

$$
(\nu, \vartheta)_{1}:=\int_{0}^{1}\left[\nu \vartheta+\nu_{x} \vartheta_{x}\right] d x, \quad \nu, \vartheta \in \mathcal{V}_{1}
$$

$A_{0}: \mathcal{V}_{1} \rightarrow \mathcal{V}_{-1}$ is given by

$$
\left(A_{0} \nu, \vartheta\right)=-\int_{0}^{1}\left[\delta_{1} \nu^{\prime}(x) \vartheta^{\prime}(x)+\delta_{2} \nu(x) \vartheta(x)\right] d x .
$$

$B_{0}: \mathbb{R} \rightarrow \mathcal{V}_{-1} \quad$ (Control operator) is given through

$$
\left(B_{0} \xi, \nu\right)=\delta_{1} \xi \nu(1), \quad \forall \xi \in \mathbb{R}, \quad \forall \nu \in \mathcal{V}_{1},
$$

i.e. $B_{0}=\delta_{1} \delta(x-1)$ is Dirac's $\delta$-function concentrated at $x=1 . C_{0}: \mathcal{V}_{0} \rightarrow \mathbb{R}$ (measurement operator) is given by

$$
C_{0} \nu:=\int_{0}^{1} k(x) \nu(x) d x, \quad \forall \nu \in \mathcal{V}_{0} .
$$

(A7): Variational solution of (40), (41)

A pair of functions $(\theta(x, t), w(t))$ is a weak solution of (38), (39) on $(0, T)$ if

$$
\begin{gathered}
\theta(\cdot, t) \in W^{1,2}(0,1), \quad w, \dot{w} \in L^{2}(0, T), \\
\int_{0}^{T}\left\{\int_{0}^{1}\left[\theta \eta_{t}-\left(\delta_{1} \theta_{x} \eta_{x}+\delta_{2} \theta \eta\right)\right] d x+\right. \\
\left.\delta_{1} \delta_{3}[\phi(t, w)+g(t)] \eta(1, t)\right\} d t=0, \\
\int_{0}^{T}\left\{w(t) \zeta(t)+\left(\int_{0}^{1} \theta(x, t) k(x) d x+\right.\right. \\
\left.\left.\delta_{4}[\phi(t, w)+g(t)]\right) \zeta(t)\right\} d t=0,
\end{gathered}
$$

$\forall$ smooth test function $\eta(x, t), \eta(x, 0)=\eta(x, 1)=0$,

$\forall$ smooth test function $\zeta(t), \zeta(0)=\zeta(T)=0$.
(A6):

Transfer function: $\chi(p)=\int_{0}^{1} \tilde{\theta}(x, p) d x$ where $\tilde{\theta}(x, p)$ is the solution of the $\operatorname{BVP}\left(k(x) \equiv 1, \delta_{3}=1, \delta_{4}=-1, \delta_{5}(t) \equiv\right.$ $\left.\delta_{5}\right)$ :

$$
\begin{aligned}
& p \tilde{\theta}=\delta_{1} \tilde{\theta}^{\prime \prime}-\delta_{2} \tilde{\theta}, \\
& \tilde{\theta}_{\left.\right|_{x=0} ^{\prime}}^{\prime}=0, \quad \tilde{\theta}_{\left.\right|_{x=1}}^{\prime}=1 . \\
\Rightarrow \quad & \tilde{\theta}(x, p)=\frac{\cosh \sqrt{p+\delta_{2}} x}{\sqrt{p+\delta_{2}} \sinh \sqrt{p+\delta_{2}}}, \\
\Rightarrow \quad & \chi(p)=\frac{1}{\sqrt{p+\delta_{2}} \sinh \sqrt{p+\delta_{2}}} \\
& \quad \int_{0}^{1} \cosh \sqrt{p+\delta_{2}} d x=\frac{1}{p+\delta_{2}}, \\
\Rightarrow & \text { sufficient to assume that }
\end{aligned}
$$

$$
|g(t)|<\frac{2}{3 \sqrt{3 \delta_{5}}} \quad \text { a.e. } t \in \mathbb{R} .
$$

$$
\begin{aligned}
& \kappa_{2}=\phi^{\prime}\left(r_{1}\right), \kappa_{3}=1 \quad \Rightarrow(\mathbf{A} 1) \\
& \chi(p)=\frac{1}{p+\delta_{2}} \\
& \lambda \in\left(0, \delta_{2}\right) \quad \Rightarrow(\mathbf{A 3}) \\
& \lambda^{2}-\delta_{2} \lambda+\kappa_{1} \leq 0 \quad \Rightarrow(\mathbf{A 6})
\end{aligned}
$$

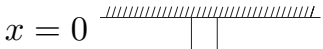

$$
\begin{aligned}
& \theta_{t}=\delta_{1} \theta_{x x}-\delta_{2} \theta \quad \longrightarrow-\delta_{2} \theta \\
& -\delta_{2} \theta \longleftarrow \quad \text { cooling } \\
& x=1 \text { A } \\
& \underbrace{w-\delta_{5} w^{3}}_{\phi(w)}+g(t)
\end{aligned}
$$

$\delta_{2}^{2} \geq 4 \kappa_{1} \Rightarrow(\mathbf{A 3})+(\mathbf{A 6})--\rightarrow$ cooling condition

\section{REFERENCES}

[1] Z. U. Blyagoz and G. A. Leonov, "Frequency criteria for stability in the large of nonlinear systems". Vestn. Leningr. Univers. 13, 18-23, 1978. (Russian)

[2] V. A. Brusin, "The Lur'e equations in Hilbert space and its solvability". Prikl. Math. Mekh. 40, 947-955, 1976. (Russian)

[3] I. M. Burkin and V. A. Yakubovich, "Frequency-domain conditions for the existence of two almost periodic solutions of a nonlinear system of automatic control". Sibirskii Mat. Zh. 16 5, 916-924, 1975. (Russian)

[4] A. G. Butkovskii, Methods for the Control of Distributed Parameter Systems. Nauka, Moscow, 1975. (Russian)

[5] Yu. A. Koryakin, G. A. Leonov, and V. Reitmann, "Konvergenz im Mittel von Phasensystemen". ZAMM 58 10, 435-441, 1978.

[6] G. A. Leonov, "On the boundedness of the trajectories of phase systems”. Sibirsk. Math. Zh. 15, 687-692, 1973. (Russian) 
[7] G. A. Leonov, V. Reitmann, and V. B. Smirnova, Non-Local Methods for Pendulum-Like Feedback Systems. B. G. Teubner Verlagsgesellschaft, Stuttgart-Leipzig, 1992.

[8] G. A. Leonov and A. N. Churilov, "Frequency-domain conditions for boundedness of solutions of phase systems". Dynamics of systems, Meshvuz. Sb., Gorky 10, 3-20, 1976. (Russian)

[9] A. L. Likhtarnikov and V. A. Yakubovich, "The frequency theorem for equations of evolutionary type". Siberian Math. J. 17, 790-803, 1976.

[10] A. Pankov, Bounded and Almost Periodic Solutions of Nonlinear Operator Differential Equations. Kluwer Academic Publishers, 1990.

[11] V. Reitmann, "Convergence in evolutionary variational inequalities with hysteresis nonlinearities". In: Proceedings of Equadiff 11, 1-10, 2005.

[12] V. Reitmann, "Über die Beschränktheit der Lösungen nichtstationärer Phasensysteme". ZAA 1, 83-93, 1982.

[13] V. Reitmann, "Existence and global stability of Bohr almost periodic solutions in evolution systems with a monotone nonlinearity". Preprint, MPI Dresden, 2006.

[14] V. Reitmann and H. Kantz, "Frequency domain conditions for the existence of almost periodic solutions in evolutionary variational inequalities". Stochastics and Dynamics 4 3, 483-499, 2004.

[15] V. A. Yakubovich, "Method of matrix inequalities in the stability theory of nonlinear control systems. Absolute stability of forced oscillations". Avtomat. Telemekh. 25, 1017-1029, 1964. (Russian) 